\title{
Erratum to: Is Notchplasty Necessary for Anatomic ACL Reconstruction?
}

Jamie Cowan, Asheesh Bedi, Hideyuki Koga, and Takeshi Muneta

\section{(C) ISAKOS 2017}

N. Nakamura et al. (eds.), Controversies in the Technical Aspects of ACL Reconstruction, DOI 10.1007/978-3-662-52742-9_25

Authorship in Chapter 25 was not correct. The correct authors of chapter 25 are Jamie Cowan, Asheesh Bedi, Hideyuki Koga, and Takeshi Muneta

The updated original online version for this chapter can be found at DOI 10.1007/978-3-662-52742-9_25

J. Cowan • A. Bedi

Sports Medicine and Shoulder Surgery,

MedSport, University of Michigan Hospitals,

Ann Arbor, MI, USA

H. Koga $\bullet$ T. Muneta $(\bowtie)$

Department of Joint Surgery and Sports Medicine, Graduate School of Medical Science, Tokyo Medical and Dental University, Tokyo, Japan

e-mail: koga.orj@tmd.ac.jp; muneta.orj@tmd.ac.jp 